\title{
Robot Mirroring: Promoting Empathy with an Artificial Agent by Reflecting the User's Physiological Affective States
}

\author{
Monica Perusquía-Hernández ${ }^{1}$, Marisabel Cuberos Balda ${ }^{2}$, David Antonio Gómez Jáuregui ${ }^{3}$, \\ Diego Paez-Granados ${ }^{4}$, Felix Dollack ${ }^{1}$ and Jose Victorio Salazar ${ }^{5}$
}

\begin{abstract}
Self-tracking aims to increase awareness, decrease undesired behaviors, and ultimately lead towards a healthier lifestyle. However, inappropriate communication of selftracking results might cause the opposite effect. Subtle selftracking feedback is an alternative that can be provided with the aid of an artificial agent representing the self. Hence, we propose a wearable pet that reflects the user's affective states through visual and haptic feedback. By eliciting empathy and fostering helping behaviors towards it, users would indirectly help themselves. A wearable prototype was built, and three user studies performed to evaluate the appropriateness of the proposed affective representations. Visual representations using facial and body cues were clear for valence and less clear for arousal. Haptic interoceptive patterns emulating heart-rate levels matched the desired feedback urgency levels with a saturation frequency. The integrated visuo-haptic representations matched to participants own affective experience. From the results, we derived three design guidelines for future robot mirroring wearable systems: physical embodiment, interoceptive feedback, and customization.
\end{abstract}

Index Terms-human-machine interaction; embodiment; empathy and intersubjectivity; haptic feedback.

\section{INTRODUCTION}

Wearable sensing technologies have enabled us to log our bodily states and behavior with the goal of monitoring our health. The number of tracked features is constantly increasing. However, its interpretation is complex. The challenge of avoiding misinterpretation remains. People might only trust information that confirms or suits their beliefs [1]. Unlike humans, machines can excel at processing high dimensional raw data. Recent machine learning techniques can be used to automatically match raw information to a humanunderstandable concept. Nevertheless, data interpretation by artificial intelligence algorithms might not be perfect and situations of distrust or over-trust might arise [2]. Thus, high levels of automation in data interpretation and user feedback should be considered carefully.

We propose quantification feedback delivered subtly, without creating awareness. The users do not necessarily need to see each measure, but rather a reflection of their health in an artificial agent. This is similar to looking at oneself in a mirror, and it would allow users to better interpret their state. In turn, this might lead to self-compassion, and trigger

\footnotetext{
${ }^{1}$ NTT Communication Science Laboratories, Japan. perusquia@ieee.org

${ }^{2}$ Independent researcher based in Switzerland.

${ }^{3}$ Univ. Bordeaux, ESTIA INSTITUTE OF TECHNOLOGY, France.

${ }^{4}$ Swiss Federal School of Technology in Lausanne (EPFL), Switzerland.

${ }^{5}$ Tohoku University, Japan.
}

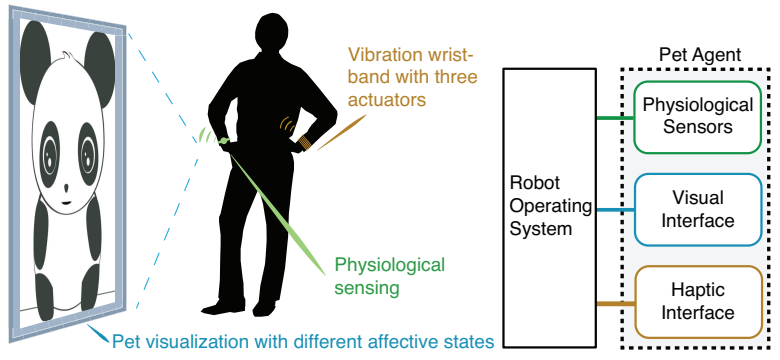

Fig. 1: Modular wearable robot mirroring framework.

healthier behaviors [3]. Deliberate mimicry has been proven as an effective strategy leading to pro-social behavior and increased liking of the mimicking person in economic [4], and courtship [5] contexts. It is also possible to foster empathy with virtual agents and robots [6], [7]. This is more noticeable in robots that are somehow perceived as weaker than the user [8]. To show caring and empathetic behavior, an agent must be attuned to the affective state of the user [9]. However, most of already implemented agents have interaction limitations due to the use of predefined dialogues. The interactions with robots tend to be more natural when adapting the robot's behavior to the user's emotional state. Anthropomorphic robots successfully show happiness, fear and neutral facial expressions as responses to the affective speech signal of users [10]. Similarly, animallike robots mimic the facial expressions and head movements of users [11], or are able to convey emotions from respiration patterns [12]. Previous work in artificial agent design has actively encouraged empathy to deal with own distress or to promote social behaviors. However, none of them explored the use of interoceptive patterns in addition to visual representations of affect, such as facial expressions.

We aim to encourage empathy towards a wearable robot, here defined as an artificial agent that senses and actuates on the user's body. The care-receiving pet mirrors and magnifies the user's state, thus providing a subtle feedback. In our implementation, the pet reflects the current user's heart rate (HR) through haptic interoceptive patterns, as a strategy to amplify the perception of the user's own body. The haptic modality is used to simulate the social effects of touch and the private nature of interoceptive sensations. Additionally, the agent represents visually different affective states mapped to the agent's facial expressions and body posture. The visual representation aims to maintain adherence over long periods of time, when compared to text-based agents [13]. A set of 
user-studies validated each component of the designed pet. Haptic feedback only, visual design only, and joint affective visuo-haptic representations. The results showed that users are able to understand and relate themselves with the agent to some extent. This set of design evaluations validated the potential to generate empathy by mirroring the user's state. In summary, the contributions of this work are:

- A framework for subtle self-tracking feedback through robot mirroring.

- A set of visuo-haptic representations of valence and arousal states using interoceptive patterns, body posture and facial expressions.

- A prototype of a modular and wearable platform for quick evaluations in the robot mirroring framework.

- An evaluation to assess the extent to which the proposed agent is able to generate empathy.

- Design recommendations for further developments.

\section{Robot MirRoring DESIGN RATIONALE}

The social needs of physical contact, empathy, compassion, and eventually caring can be projected into a pet robot that mirrors the users themselves. The following design requirements should be considered: (1) the robot should be able to mirror several levels of the user's health state to trigger empathy; (2) the robot should appear as weaker than the user to foster care-receiving treats; (3) the robot should measure the user's state through several sensors arranged in a comfortable wearable; and (4) the data obtained should be processed to roughly estimate the user's state. These requirements were translated into our concept as follows:

To represent and mirror affective states, we chose visuo-haptic representations based on a mapping of posture, facial expressions, and HR to the circumplex model of affect [14]. Two dimensions, valence and arousal, are used to represent emotions. Valence corresponds to the degree of pleasantness, from negative to positive. Arousal represents the level of alertness or activation. Visual representations of both valence and arousal are usually successful [13]; and wearable devices can be used to produce vibration patterns that can be recognized by the user in ecological settings [15].

To measure the user's state, we chose easy-to-wear autonomic physiological measures such as Heart Rate (HR) derived from Blood Volume Pulse (BVP). It has been suggested that changes in our body states create the subjective feeling of an emotion [16]. Facial expressions are a good predictor of the valence [17]. Other autonomic physiological changes are often associated with arousal [18] and with emotional responses to an event or to an interaction with another agent [19]. BVP is relevant for estimation of affective states [20]. Moreover, wearable devices can serve as both a sensor and as biofeedback device to convey emotions by breathing patterns [21]. In addition, small pet robots can also convey arousal levels through breathing patterns [12]. Nevertheless, we argue that using heart-rate is a more robust feedback method than breathing, as it cannot be controlled voluntarily. Finally, physiological measurement devices are private to the user. They record only the person who chose to wear the device, and can be hidden under other garments.

To foster empathy, we opted to enhance interoceptive cues such as heart beat. Interoception is defined as the ability to correctly perceive bodily sensations [22]. The ability to perceive our own affective states might be linked to the empathy we feel for another. Mimicking the behaviors of others, and physiological synchronization is related to higher empathetic accuracy [23], [24]. In addition, user's physiological cues (heartbeat) associated to agents can enhance their engagement [25]. Because of this, enhancement of our own affective states might be a necessary element to increase empathy. Furthermore, high interoceptive awareness has been linked to a greater automatic imitation [26]. Interoceptive cues can be represented using haptic patterns in a private and unobtrusive manner.

To promote care-receiving behaviors, we encourage personalization to create a sense of ownership and responsibility for the pet, plus a weak appearance. The pet was assigned according to the user's personality to foster ingroup feelings. The users also customized their pet to create appreciation by spending some time modifying it [27].

Therefore, we propose a modular wearable robot with both sensing and actuation (fig. 1). We used one wristband for biosignal measurement and a second wristband integrated with three vibration actuators. Finally, we presented a visualization of the pet on a screen. We conducted separate design iterations and evaluations for each element.

\section{VISUAL REPRESENTATION}

A pet agent was designed to fit nine zones of the circumplex model of affect. These zones were all combinations of three arousal levels (low, neutral and high) and three valence levels (low, neutral and high). The pet was a caricaturized antropomorphic character as reflected in the pet's ability to show human-like affective displays. This "social activation model" contributes to foster empathy [28]. Two iterative online surveys were used to (1) refine the visualizations, and (2) ensure that each visual representation conveys a specific emotion to the user (fig. 2).

\section{A. Methods}

1) Participants: 115 participants took part in the first iteration (64 female, average age $=33.74 \pm 9.29$ years old). 32 participants ( 16 female, average age $=31.84 \pm 5.45$ years old) took part in the second iteration.

2) Procedure: The pet representations were presented in random order, together with the question "How is the character in the image feeling?" answered with the Affect Grid (AG) [29]. After rating the nine images, demographic questions were asked.

3) Results: We expected the densities to be centered around the intended values (-4, 0, 4 per dimension) for each facial expression of the pet. However, the representation of arousal was not clear. Hence, body movement indications were added. Low arousal was represented by a panda sitting or lying down and with drowsy eyes. High arousal was 


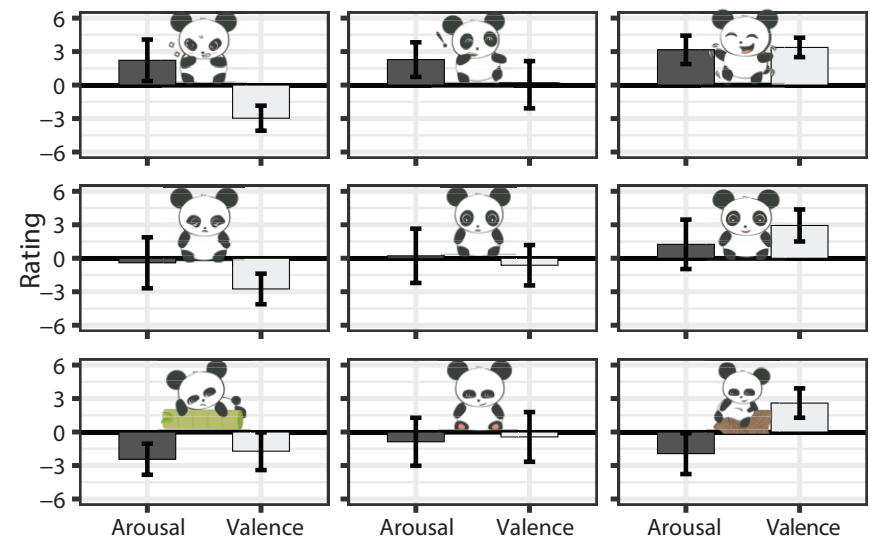

Fig. 2: Pet visualizations along the valence and arousal circumplex, for the second round of online survey rating results.

depicted by movement of the limbs such as lifting and bending. The second iteration showed more focused distributions, except for the cases with neutral valence. This seems in line with previous research that suggested that even neutral faces are appraised positively or negatively according to the context or the personality of the rater [30].

\section{HAPTIC REPRESENTATION}

Embodied haptic feedback was designed to convey different arousal levels with a train of vibration pulses resembling a beating heart. Higher HRs are expected to be perceived as higher arousal levels. The user's HR was used as baseline. An evaluation study was conducted to assess the affective meaning assigned to the proposed vibration patterns and the level of urgency they communicate.

\section{A. System implementation}

The HR of each user was mapped to a vibration pattern simulating the heart sounds $S_{1}$ and $S_{2}$ which correspond to the QRS complex and T-wave of the heart's electrical activity [31]. The pattern was produced three times, representing three cardiac cycles. The time for each pulse was fixed to $t_{\text {base }}=100 \mathrm{~ms}$ and the time between the start of each pattern is calculated from the user's HR as $t_{r e p}=60 / H R_{i n}$. The time between $S_{1}$ and $S_{2}, t_{Q-T}$ was set as $15 \%$ of $t_{r e p}$, where $H R_{i n}=B P M * x$ is one of four interoceptive patterns, calculated by multiplying the user's base HR by several factors: $x=(0.5,1,2,4)$.

The wristband was made with flexible material $(40 \%$ rubber, $30 \%$ acrylic, $30 \%$ polyester) and three Xiaomi Mi 2 fitness band cores (fig. 3a). The stitches were minimal to reduce bumps in the fabric while keeping the fitness cores in place. The fitness bands have a coin vibration motor used for alerting the wearer, and connect via Bluetooth to a laptop NEC Lavie Hz750/C running the Robot Operating System (ROS) Kinetic on Ubuntu 16.04 LTS. A custom Python script to control the vibrations was written based on [32] and [33]. EDA and BVP were measured using an Empatica e4 and the iOS E4 realtime App on an iPhone SE. Measurements were synchronized using the button on the Empatica. The vibration patterns were triggered manually two seconds after inserting a marker in the Empatica data.

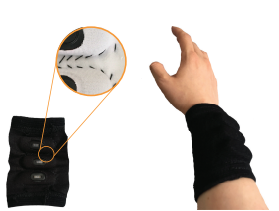

(a) Elastic band designed with three vibration actuators.

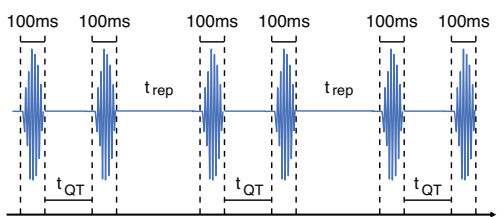

(b) Urgency levels feedback. $t_{Q T}$ is the time between vibrations imitating a cardiac cycle. $t_{r e p}$ is the time between cardiac cycles.
Fig. 3: Haptic wearable and vibrotactile feedback.

\section{B. Methods}

The haptic feedback was tested in two iterations according to the feedback obtained from an on-site HTML survey.

1) Participants: 36 participants took part in the study (20 female, average age $=29.54 \pm 8.21$ years old).

2) Procedure: First, the participants were asked to wear the wristband and an Empatica e4. Then they reported their feelings at that moment with the AG. Next, they experienced the vibration patterns in random order, and rated them using (1) the AG, and (2) a VAS scale reporting the level of urgency that the pattern made them feel.

3) Analysis and results: The relationship between the number of beats and the level of urgency was proportional (fig. 47. A non-parametric Friedman test of references among repeated measures was conducted and rendered a significant Chi-square value of $40.96(p<0.01)$. A pair-wise sign test indicated that the participants could tell the difference between patterns 1,2,3; and between 1-4 $(p<0.01)$; but could not tell the difference between 2-4 $(p=0.12)$ and 3-4 $(p=1)$. The high frequency in the $\mathrm{x} 4$ pattern reached a saturation point in perception, where individual patterns were not recognizable anymore; i.e., they were perceived as single vibrations. A one sample t-test showed that the valence was consistently rated as neutral $(M=0, t(143)=-5.07$, $p<.001,95 \%$ CI [-.59 .02], $d=-0.29)$. As expected from the urgency ratings, arousal ratings were proportional to the multiplying HR factor. An ANOVA with the AG ratings as dependent variable, and the interactions between pattern and AG axis (valence and arousal) was performed. The results yielded significant differences between the different patterns $(F(3,276)=5.27, p<.01)$, and the interaction between patterns and the AG axis $(F(3,276)=13.764, p<.001)$. Tukey HSD pair-wise comparisons showed no significant differences for arousal levels in patterns 1-2 $(p=0.10)$ and 2-3 $(p=0.53)$; and significant differences in pairs 1-3, 1$4,2-4$, and 3-4 $(p<.001)$. These results suggest that the desired interpretation of the interoceptive patterns is correctly communicated. Pattern 1 is expected to be less arousing than the neutral (baseline state). Patterns 2 to 4 are designed to have an increasing arousal interpretation. The only pattern that fails to do so is pattern 4 , as it was perceived as it reaches saturation of haptic perception, and therefore is perceived as even less arousing than pattern 1. Hence, the maximum multiplicative factor that can be used for this type of haptic feedback is between $\mathrm{x} 2$ and $\mathrm{x} 4$. 


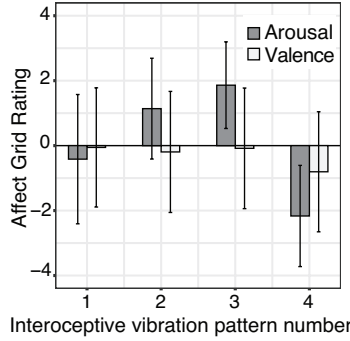

(a) Ratings of arousal and valence per vibration pattern.

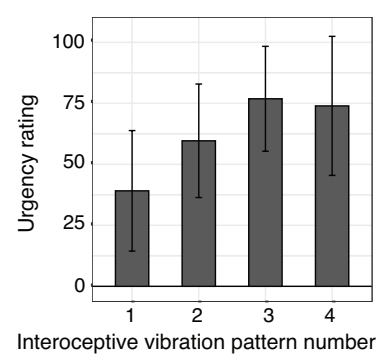

(b) Urgency levels per vibration pattern.
Fig. 4: Haptic evaluation results. Error bars indicate SD.

\section{Modality Integration AND EMPathiC EVALUATION}

Visual representations and interoceptive patterns were integrated in a single stimuli. The pet expressions were animated to go from a neutral state to the apex. The haptics were started together with the animation. The interoceptive patterns were mapped to distinct levels of arousal. High levels of arousal were represented by faster HR, low arousal levels were communicated with slower HR. Additionally, a simple personalization task was designed to foster empathy with the pet in a short interaction. This task included providing a "tailor-made" pet that was later named by the users.

\section{A. System implementation}

The prototype used for the haptic evaluation was improved. The physiological data logging, and the vibration triggering were automatized and synchronized with the visualizations. The vibration pattern was updated in real-time with the HR values as measured from the Empatica e4 (fig. 11. The Empatica data was broadcasted to ROS with the Empatica Android SDK v2.2 and rosjava. To broadcast the Empatica's data, the android sample project from Empatica was integrated with the ROS Android sample app based on rosjava [34]. The Empatica-measured values for BVP were sent in a custom ROS message [35] and published in a topic. An HTML form was used to present the user with the visual stimuli. This form sends vibration initiation commands to ROS via JavaScript (JS) and a rosbridge server. The vibration frequency is modulated by a factor $(0.5,1,2)$ depending on (1) the user's HR taken as baseline; and (2) the expected emotion induced from the videos shown in the HTML form (0.5 was assigned to low arousal, 1 to neutral arousal, and 2 to high arousal). Finally, the vibration pattern is executed by an additional ROS topic, triggered by the HTML form and using the published Empatica-measurements.

\section{B. Methods}

1) Participants: 23 volunteers took part in the study (10 female, average age $=27.2 \pm 7.44$ years old).

2) Experiment design: The goal was to investigate whether congruent, mirroring reactions could influence the closeness felt by the participants with the pet. A 2 (match) x 9 (emotion) mixed design was used. Nine videos of different valence and arousal were presented in two groups. Participants assigned to the matching condition saw a pet representation that matched the valence and arousal of the video they just watched. Participants in the mismatching condition had contact with pets which represented a different feeling than the one conveyed by the video that they just watched.

3) Stimuli: Emotion-eliciting film scenes from two publicly available databases were chosen: the FilmStim database [36] and the Emotional Movie Database (EMDB) [37]. These databases have already validated the main emotion conveyed with each stimuli. Similarly to the visual representations, the nine videos were selected to match low, neutral and high valence and arousal levels, and to match each one of the nine pet visualizations (table $\mathbb{I}$ ). For high arousal, and neutral and positive valence, we decided to use stimuli suitable for minors instead of the database stimuli.

TABLE I: Emotion-eliciting video sequences representing each pet visualization. V: valence. A: arousal. L: low, $\mathrm{N}$ : neutral, $\mathrm{H}$ : high.

\begin{tabular}{cll}
\hline $\begin{array}{c}\text { Affect Grid } \\
\text { Values }\end{array}$ & Corresponding video sequence name & $\begin{array}{l}\text { Video } \\
\text { source }\end{array}$ \\
\hline N-V, N-A & Blue & FilmStim [36] \\
N-V, L-A & 6000 & EMDB [37] \\
L-V, N-A & E.T. & FilmStim [36] \\
H-V, N-A & The Dead Poets society & FilmStim [36] \\
HL-V, L-A & Life is beautiful (La vita e bella) & FilmStim [36] \\
N-V, H-A & Wiserhood & {$[38]$} \\
H-V, H-A & Baby laughing hysterically at ripping paper & {$[39]$} \\
L-V, H-A & Schindler's list & FilmStim [36] \\
L-V, L-A & City of angels & FilmStim [36] \\
\hline
\end{tabular}

4) Procedure: The participants were explained the procedure and signed an informed consent. Following an automatized HTML page, they answered the State-Trait Anxiety Inventory (STAI-Y2) questionnaire [40], and were informed by the system that a pet was automatically selected based on their answers. They were asked to name it. Then the pet got happy and celebrated their new name. The STAI questionnaire was only used to make the participants believe that the pet was created from their answers to this questionnaire. In reality, the same pet was used for all participants. This was chosen to boost their sense of ownership and to foster a sense of group belonging with the pet. Next, participants were asked to watch the aforementioned nine emotion elicitation videos. After each video, they were asked to report their affective state using the AG. Next, the pet appeared along with the respective interoceptive vibration pattern calculated in real-time. After watching the pet animation, they were asked to report how the pet felt based on the AG. After all nine stimuli were watched, participants were asked to answer the Inclusion of Other in the Self (IOS) scale [41]. This scale ranges from 1 (low) to 7 (high), measuring how close they think the pet is with themselves. Next, demographic questions were asked. Finally, a semi-structured interview was conducted to investigate what and why their impressions were on the pet visualization, the haptic feedback, and the robot mirroring concept.

5) Analysis and results: The valence ratings for the visuohaptic representations were somewhat consistent, independently of the experimental condition (fig. 5a. Wilcoxon rank sum test $V=5396.5, p=0.91)$. Arousal ratings differed 


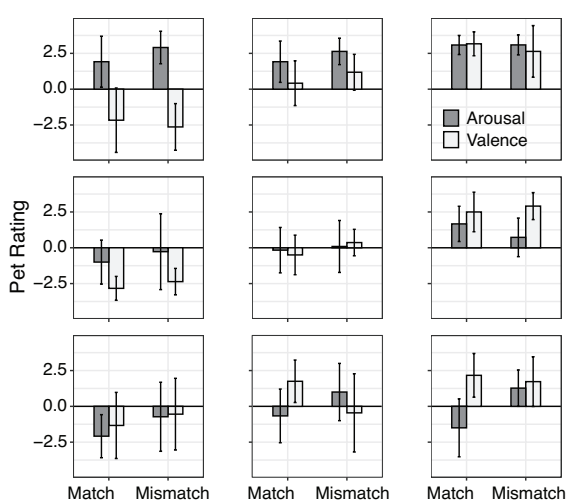

(a) Rating of the pet's feelings per match and mismatch experimental conditions
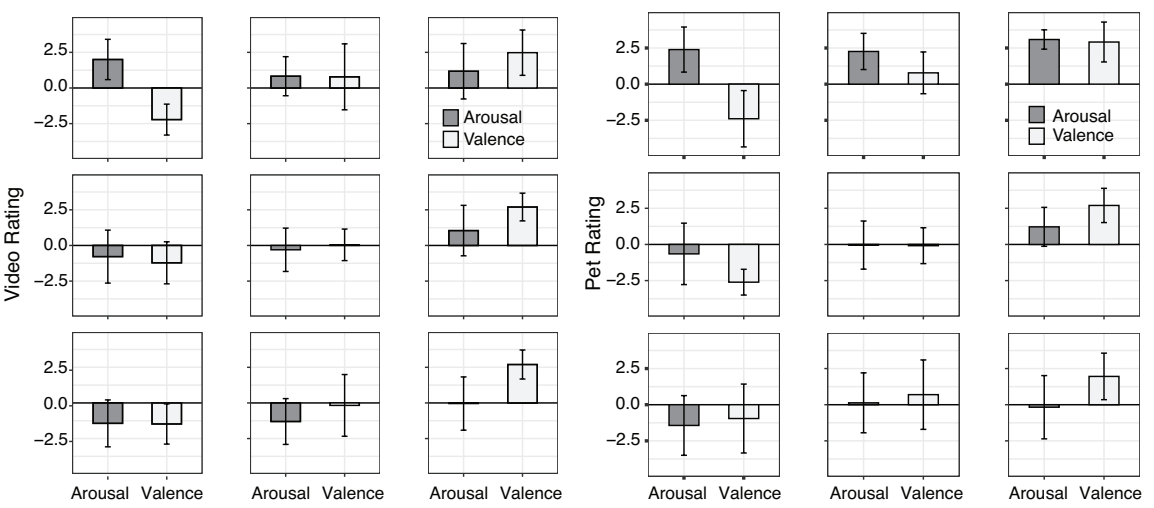

(b) Self-report after watching each stimuli video

(c) Rating of the pet's feelings regardless of experimental condition

Fig. 5: Results of the sensitizing evaluation. Error bars indicate SD.

in match and mismatch conditions $(V=4220, p<0.01)$. This is probably due to the baseline changes for the interoceptive vibrations, following the HR of the user and their arousal state induced from the previously watched video. As expected, arousal and valence ratings are consistent with each video and pet visual stimuli (Fig. 5b, 5c). To confirm that the arousal perception depends on the user's state, an ANOVA with affective rating as dependent variable and the experimental condition and stimuli type (video or pet) as independent variables was performed. No significant differences were found between experimental conditions $(F(1,824)$ $=1.25, p=.26)$, stimuli types $(F(1,824)=3.06, p=.81)$, or the interactions between them $(F(1,824)=1.96, p=.16)$. The average IOS scores were $3.42(\mathrm{SD}=1.32)$ for the matching condition, and $2.82(\mathrm{SD}=1.90)$ for the mismatching condition. A Kruskal-Wallis rank sum test found no difference in IOS ratings per experimental conditions (Chi-squared $=1.1498, \mathrm{df}=1, \mathrm{p}=.28)$. This suggests that, at least for this design, the level of closeness with the pet is independent from the matching behavior of the pet. Additionally, the participants verbal reports were analyzed using affinity diagrams. According to the results, the participants perceived that the pet was somehow linked to their own emotion: "The panda felt similar than me, but it was more expressive."-P4, Male, 29 yo. Nevertheless, the IOS scores are rather modest and leave significant room for improvement. Several participants expressed their preference to choose their own pet, instead of it being assigned to them. Interestingly, Chinese nationals $(\mathrm{N}=3)$ were happy with the panda choice, as they already felt identified with this animal. Two participants mentioned that a virtual pet cannot compare to (their) real pets. Another participant mentioned that the characterization of the virtual character might make it more suitable for children, and that a challenge might be to maintain long-term engagement ( $\mathrm{P} 7$, male, 31 yo). Finally, one participant expressed his wish to have the robot mirroring pet to give smart personalized recommendations of activities that would bring him into a positive mood without him having to look for them in social media or emails (P1, male, 26 yo).

\section{DISCUSSION AND IMPLICATIONS FOR DESIGN}

The main purpose of this work was to test the mirroring concept for subtle self-tracking feedback by fostering empathy with a wearable robot representing the self. Mirroring of the user's state is achieved through visuohaptic representations including facial expression, posture, and interoceptive factors. The first evaluation conducted with two online surveys showed that participants were able to accurately infer the intended affective valence message from the visual representations. The second evaluation aimed to assess the understanding of the proposed interoceptive haptic feedback. The participants were able to relate the heart rate to different levels of urgency in the embodied device (arousal levels) with a saturation point. We also confirmed that the integrated visuo-haptic version accurately conveyed the intended affective state. The relationship of the the pet representation and the users' own affective experience ratings hints to subtle empathy between the users and the pet.

Despite the success of the chosen representations, the closeness ratings with the pet could be further improved. Pet personalization should include more pet types; and adjust the range of emotions based on the personality of the user (e.g. a more shy or extroverted pet). The synchronization between haptic and visual modalities could also be improved by including interoceptive feedback in the visuals with pet respiration animations to increase haptic salience and avoid any misinterpretation of the haptic feedback.

Based on our results, we suggest three recommendations for future robot mirroring designs:

1) Physical embodiment. Our results suggest that visual representations are good enough to represent different combinations of arousal and valence. These should systematically vary eye opening, body posture, and mouth shape. However, tangibility of the pet is important to foster care-receiving behaviors. Visuo-haptic time synchronization must be performed according to interoceptive patterns to increase agent salience.

2) Interoceptive feedback. Interoception enhancement through haptic feedback is an intuitive manner of communicating arousal levels in a discrete and embodied manner. The HR amplification has a saturation level at 
multiplying factors of more than three.

3) Customization. Personality-adjusted pet expressiveness and choice of the pet type would improve the closeness between the user and the pet.

\section{ACKNOWLEDGMENTS}

We thank Els van Dijk, Aisen Caro Chacin and Ryoichiro Shiraishi for early comments on the concept; and Miwa Saito for her support conducting some of the experiments.

\section{REFERENCES}

[1] J. Klayman, "Varieties of Confirmation Bias," Psychology of Learning and Motivation, vol. 32, pp. 385-418, jan 1995.

[2] E. T. van Dijk, F. Beute, J. H. Westerink, and W. A. Ijsselsteijn, "Unintended effects of self-tracking," CHI'15, Workshop on Beyond Personal Informatics: Designing for Experiences of Data, p. 5, 2015.

[3] K. Neff, "Self-Compassion: An Alternative Conceptualization of a Healthy Attitude Toward Oneself," Self and Identity, vol. 2, no. 2, pp. 85-101, apr 2003.

[4] R. B. van Baaren, R. W. Holland, B. Steenaert, and A. van Knippenberg, "Mimicry for money: Behavioral consequences of imitation," Journal of Experimental Social Psychology, vol. 39, no. 4, pp. 393398, jul 2003.

[5] N. Guéguen, "Mimicry and seduction: An evaluation in a courtship context," Social Influence, vol. 4, no. 4, pp. 249-255, oct 2009.

[6] R. S. Rosenberg, S. L. Baughman, and J. N. Bailenson, "Virtual superheroes: Using superpowers in virtual reality to encourage prosocial behavior," PLOS ONE, vol. 8, no. 1, pp. 1-9, 012013.

[7] A. Cordar, M. Borish, A. Foster, and B. Lok, "Building virtual humans with back stories: Training interpersonal communication skills in medical students," in Intelligent Virtual Agents, T. Bickmore, S. Marsella, and C. Sidner, Eds. Cham: Springer International Publishing, 2014, pp. $144-153$.

[8] F. Tanaka, A. Cicourel, and J. R. Movellan, "Socialization between toddlers and robots at an early childhood education center." Proceedings of the National Academy of Sciences of the United States of America, vol. 104, no. 46, pp. 17954-8, nov 2007.

[9] R. Picard, Affective Computing. Cambridge, MA: MIT Press, 1997.

[10] F. Hegel, T. Spexard, B. Wrede, G. Horstmann, and T. Vogt, "Playing a different imitation game: Interaction with an Empathic Android Robot," in Proceedings of the 2006 6th IEEE-RAS International Conference on Humanoid Robots, HUMANOIDS, 2006, pp. 56-61.

[11] L. D. Riek, P. C. Paul, and P. Robinson, "When my robot smiles at me: Enabling human-robot rapport via real-time head gesture mimicry," Journal on Multimodal User Interfaces, vol. 3, no. 1, pp. 99-108, mar 2010.

[12] P. Bucci, L. Cang, A. Valair, D. Marino, L. Tseng, M. M. Jung, J. Rantala, O. S. Schneider, and K. E. MacLean, "Sketching cuddlebits: Coupled prototyping of body and behaviour for an affective robot pet," in CHI '$^{\prime} 17,2017$.

[13] S. Provoost, J. Ruwaard, K. Neijenhuijs, T. Bosse, and H. Riper, "Mood Mirroring with an Embodied Virtual Agent: A Pilot Study on the Relationship Between Personalized Visual Feedback and Adherence," in Highlights of Practical Applications of Agents, Multi-Agent Systems, and Complexity: The PAAMS Collection. Springer, Cham, jun 2018, pp. 24-35.

[14] J. A. Russell, "A circumplex model of affect," Journal of Personality and Social Psychology, vol. 39, no. 6, pp. 1161-1178, 1980.

[15] J. R. Cauchard, J. L. Cheng, T. Pietrzak, and J. A. Landay, "Ambivibe: Design and evaluation of vibrations for progress monitoring," Proceedings of the 2016 CHI Conference on Human Factors in Computing Systems, 2016.

[16] W. James, "What is an emotion?" Mind, vol. os-IX, no. 34, pp. 188205, apr 1884.

[17] J. T. Cacioppo, R. E. Petty, M. E. Losch, and H. S. Kim, "Electromyographic activity over facial muscle regions can differentiate the valence and intensity of affective reactions." Journal of personality and social psychology, vol. 50, no. 2, pp. 260-8, feb 1986.

[18] S. D. Kreibig, "Autonomic nervous system activity in emotion: a review," Biological Psychology, vol. 84, no. 3, pp. 394-421, jul 2010.
[19] G. Chanel and C. Mühl, "Connecting brains and bodies: Applying physiological computing to support social interaction," Interact. Comput., vol. 27, pp. 534-550, 2015

[20] T. Kameda, A. Murata, C. Sasaki, S. Higuchi, and K. Inukai, "Empathizing With a Dissimilar Other," Personality and Social Psychology Bulletin, vol. 38, no. 8, pp. 997-1003, aug 2012.

[21] J. Frey, M. Grabli, R. Slyper, and J. R. Cauchard, "Breeze: Sharing biofeedback through wearable technologies," Proceedings of the 2018 CHI Conference on Human Factors in Computing Systems, 2018.

[22] G. Zamariola, P. Maurage, O. Luminet, and O. Corneille, "Interoceptive accuracy scores from the heartbeat counting task are problematic: Evidence from simple bivariate correlations," Biological Psychology, vol. 137, pp. 12-17, sep 2018.

[23] J. A. Soto and R. W. Levenson, "Emotion Recognition across Cultures: The Influence of Ethnicity on Empathic Accuracy and Physiological Linkage," Emotion (Washington, D.C.), vol. 9, no. 6, p. 874, 2009.

[24] R. W. Levenson and A. M. Ruef, "Empathy: A Physiological Substrate," Journal of Personality and Social Psychology, vol. 63, no. 2, pp. 234-246, 1992.

[25] J. Frey, "Heart rate monitoring as an easy way to increase engagement in human-agent interaction," ArXiv, vol. abs/1412.1772, 2015.

[26] V. Ainley, M. Brass, and M. Tsakiris, "Heartfelt imitation: High interoceptive awareness is linked to greater automatic imitation," Neuropsychologia, vol. 60, pp. 21-28, jul 2014.

[27] M. I. Norton, D. Mochon, and D. Ariely, "The IKEA effect: When labor leads to love," Journal of Consumer Psychology, vol. 22, no. 3, pp. 453-460, jul 2012.

[28] J. Fink, "Anthropomorphism and human likeness in the design of robots and human-robot interaction," in Lecture Notes in Computer Science (including subseries Lecture Notes in Artificial Intelligence and Lecture Notes in Bioinformatics), vol. 7621 LNAI. Springer, Berlin, Heidelberg, oct 2012, pp. 199-208.

[29] J. A. Russell, A. Weiss, and G. A. Mendelsohn, "Affect Grid: A SingleItem Scale of Pleasure and Arousal," Journal of Personality and Social Psychology, vol. 57, no. 3, pp. 493-502, 1989.

[30] B. Meyer, P. A. Pilkonis, and C. G. Beevers, "What's in a (neutral) face? Personality disorders, attachment styles, and the appraisal of ambiguous social cues," Journal of Personality Disorders, vol. 18, no. 4, pp. 320-336, 2004.

[31] S. Salleh, S. H. S. Hussain, T. S. Tan, C.-M. Ting, A. Noor, S. Pipatsart, J. Ali, and P. Yupapin, "Acoustic cardiac signals analysis: A kalman filter-based approach," International journal of nanomedicine, vol. 7, pp. 2873-81, 062012.

[32] "creotiv/MiBand2: Library to work with Xiaomi MiBand 2," 2018. [Online]. Available: https://github.com/creotiv/MiBand2

[33] J. Salazar and Y. Hirata, "Customizable Vibrotactile Feedback using Network of Independent Vibration Devices in ROS," in 37th Annual Conference of the Robotics Society of Japan, 2019.

[34] "ROS E4 link via Android," 2019. [Online]. Available: https: //github.com/MonicaPH/ros \{_\}android \{_\}e4

[35] "Empatica ROS Java messages," 2018. [Online]. Available: https: //github.com/hyeonukbhin/empatica $\left\{{ }_{-}\right\} \mathrm{e} 4\left\{{ }_{-}\right\} \mathrm{msgs}$

[36] A. Schaefer, F. Nils, X. Sanchez, and P. Philippot, "Assessing the effectiveness of a large database of emotion-eliciting films: A new tool for emotion researchers," Cognition and Emotion, vol. 24, no. 7, pp. 1153-1172, 2010.

[37] S. Carvalho, J. Leite, S. Galdo-Álvarez, and Ó. F. Gonçalves, "The emotional movie database (emdb): A self-report and psychophysiological study," Applied Psychophysiology and Biofeedback, vol. 37, no. 4, pp. 279-294, Dec 2012.

[38] Wiserhoode, "JP Wiser's Rye TV Commercial, 'Purse')," 2014. [Online]. Available: https://www.ispot.tv/ad/7TCX/jp-wisers-rye-purse

[39] BruBearBaby, "Baby Laughing Hysterically at Ripping Paper (Original)," 2011. [Online]. Available: https://www.youtube.com/ watch? $=$ RP4abiHdQpc

[40] C. D. Spielberger, R. L. Gorsuch, R. Lushene, P. R. Vagg, and G. A. Jacobs, Manual for the State-Trait Anxiety Inventory. Palo Alto, CA: Consulting Psychologists Press, 1983.

[41] A. Aron, E. N. Aron, and D. Smollan, "Inclusion of Other in the Self Scale and the structure of interpersonal closeness." Journal of Personality and Social Psychology, vol. 63, no. 4, pp. 596-612, 1992. 